\title{
ON GROUPS WITH THE SAME CHARACTER DEGREES AS ALMOST SIMPLE GROUPS WITH SOCLE THE MATHIEU GROUPS
}

\author{
SEYED HASSAN ALAVI, ASHRAF DANESHKHAH, AND ALI JAFARI
}

\begin{abstract}
Let $G$ be a finite group and $\operatorname{cd}(G)$ denote the set of complex irreducible character degrees of $G$. In this paper, we prove that if $G$ is a finite group and $H$ is an almost simple group whose socle is Mathieu group such that $\operatorname{cd}(G)=\operatorname{cd}(H)$, then there exists an Abelian subgroup $A$ of $G$ such that $G / A$ is isomorphic to $H$. This study is heading towards the study of an extension of Huppert's conjecture (2000) for almost simple groups.
\end{abstract}

\section{INTRODUCTION}

Let $G$ be a finite group, and let $\operatorname{Irr}(G)$ be the set of all complex irreducible character degrees of $G$. Denote by $\operatorname{cd}(G)$ the set of character degrees of $G$, that is to say, $\operatorname{cd}(G)=\{\chi(1) \mid \chi \in \operatorname{Irr}(G)\}$. It is well-known that the complex group algebra $\mathbb{C} G$ determines character degrees and their multiplicities.

There is growing interest in a question regarding the structure of $G$ which can be recovered from the character degree set of $G$ with or without multiplicity. It is well-known that the character degree set of $G$ can not use to completely determine the structure of $G$. For example, the non-isomorphic groups $D_{8}$ and $Q_{8}$ not only have the same set of character degrees, but also share the same character table. The character degree set cannot be used to distinguish between solvable and nilpotent groups. For example, if $G$ is either $Q_{8}$ or $S_{3}$, then $\operatorname{cd}(G)=\{1,2\}$. Recently, Navarro [14] showed that the character degree set alone cannot determine the solvability of the group. Indeed, he constructed a finite perfect group $H$ and a finite solvable group $G$ such that $\operatorname{cd}(G)=\operatorname{cd}(H)$. It is also discovered by Navarro and Rizo [15] that there exists a finite perfect group and a finite nilpotent group with the same character degree set. Notice that in both examples, these finite perfect groups are not nonabelian simple. It remains open whether the complex group algebra can determine the solvability of the group or not (see Brauer's Problem 2 [5]).

However, the situation for simple groups and related groups is rather different. It has been proved recently that all quasisimple groups are uniquely determined up to isomorphism by their complex group algebras [3] in which a finite group $G$ is quasisimple if $G$ is perfect and $G / Z(G)$ is a nonabelian simple group. In the late 1990s, Huppert [10] posed a conjecture which asserts that the nonabelian simple groups are essentially characterized by the set of their character degrees.

Date: September 19, 2018.

2010 Mathematics Subject Classification. Primary 20C15; Secondary 20D05.

Key words and phrases. Character degrees; Almost simple groups; Sporadic simple groups; Mathieu groups; Huppert's Conjecture.

Corresponding author: Ashraf Daneshkhah. 
Conjecture 1.1. (Huppert) Let $G$ be a finite group and $H$ a finite nonabelian simple group such that the sets of character degrees of $G$ and $H$ are the same. Then $G \cong H \times A$, where $A$ is an abelian group.

This conjecture is verified for Alternating groups, many of the simple groups of Lie type $[16,19,20]$ and for all sporadic simple groups $[1,2,11,18]$.

In this paper, we initiate an investigation on an extension of Huppert's conjecture for almost simple groups whose socle is the sporadic simple groups. A group $H$ is called almost simple if there exists a nonabelian simple group $H_{0}$ such that $H_{0} \leqslant$ $H \leqslant \operatorname{Aut}\left(H_{0}\right)$. Indeed, this paper is devoted to studying finite groups with the same character degrees as almost simple groups $H$ with socle $H_{0}$ being one of the Mathieu groups:

Theorem 1.1. Let $G$ be a finite group, and let $H$ be an almost simple group whose socle is one of the Mathieu groups. If $\operatorname{cd}(G)=\mathrm{cd}(H)$, then there exists an abelian group $A$ such that $G / A$ is isomorphic to $H$.

In order to prove Theorem 1.1, we establish the following steps which Huppert introduced in [10]. Let $H$ be an almost simple group with socle $H_{0}$, and let $G$ be a group with the same character degrees as $H$. Then we show that

Step 1.: $G^{\prime}=G^{\prime \prime}$;

Step 2.: if $G^{\prime} / M$ is a chief factor of $G$, then $G^{\prime} / M$ is isomorphic to $H_{0}$;

Step 3.: if $\theta \in \operatorname{Irr}(M)$ with $\theta(1)=1$, then $I_{G^{\prime}}(\theta)=G^{\prime}$ and so $M=M^{\prime}$;

Step 4.: $M=1$ and $G^{\prime} \cong H_{0}$;

Step 5.: $G / C_{G}\left(G^{\prime}\right)$ is isomorphic to $H$.

Note that to prove Step 2, we determine all finite simple groups whose irreducible character degrees divide some irreducible character degrees of almost simple groups with socle sporadic simple groups, and by Proposition 3.1, all such simple groups are listed in Table 1. This result somehow relates to [17, Theorem 1] for sporadic simple groups.

\section{Preliminaries}

In this section, we present some useful results to prove Theorem 1.1. We first establish some definitions and notation.

Throughout this paper all groups are finite. Recall that a group $H$ is said to be an almost simple group with socle $H_{0}$ if $H_{0} \leqslant H \leqslant$ Aut $\left(H_{0}\right)$, where $H_{0}$ is a nonabelian simple group. For a positive integer $n, \pi(n)$ denotes the set of all prime divisors of $n$. If $G$ is a group, we will write $\pi(G)$ instead of $\pi(|G|)$. If $N \unlhd G$ and $\theta \in \operatorname{Irr}(N)$, then the inertia group of $\theta$ in $G$ is denoted by $I_{G}(\theta)$ and is defined by $I_{G}(\theta)=\left\{g \in G \mid \theta^{g}=\theta\right\}$. If the character $\chi=\sum_{i=1}^{k} e_{i} \chi_{i}$, where each $\chi_{i}$ is an irreducible character of $G$ and $e_{i}$ is a nonnegative integer, then those $\chi_{i}$ with $e_{i}>0$ are called the irreducible constituents of $\chi$. The set of all irreducible constituents of $\theta^{G}$ is denoted by $\operatorname{Irr}(G \mid \theta)$. All further notation and definitions are standard and could be found in $[9,12]$.

Lemma 2.1. [9, Theorems 19.5 and 21.3] Suppose $N \unlhd G$ and $\chi \in \operatorname{Irr}(G)$.

(a) If $\chi_{N}=\theta_{1}+\theta_{2}+\cdots+\theta_{k}$ with $\theta_{i} \in \operatorname{Irr}(N)$, then $k$ divides $|G / N|$. In particular, if $\chi(1)$ is prime to $|G / N|$, then $\chi_{N} \in \operatorname{Irr}(N)$.

(b) (Gallagher's Theorem) If $\chi_{N} \in \operatorname{Irr}(N)$, then $\chi \psi \in \operatorname{Irr}(G)$ for all $\psi \in \operatorname{Irr}(G / N)$. 
Lemma 2.2. [9, Theorems 19.6 and 21.2] Suppose $N \unlhd G$ and $\theta \in \operatorname{Irr}(N)$. Let $I=I_{G}(\theta)$.

(a) If $\theta^{I}=\sum_{i=1}^{k} \phi_{i}$ with $\phi_{i} \in \operatorname{Irr}(I)$, then $\phi_{i}^{G} \in \operatorname{Irr}(G)$. In particular, $\phi_{i}(1) \mid G$ : $I \mid \in \operatorname{cd}(G)$.

(b) If $\theta$ extends to $\psi \in \operatorname{Irr}(I)$, then $(\psi \tau)^{G} \in \operatorname{Irr}(G)$ for all $\tau \in \operatorname{Irr}(I / N)$. In particular, $\theta(1) \tau(1)|G: I| \in \operatorname{cd}(G)$.

(c) If $\rho \in \operatorname{Irr}(I)$ such that $\rho_{N}=e \theta$, then $\rho=\theta_{0} \tau_{0}$, where $\theta_{0}$ is a character of an irreducible projective representation of $I$ of degree $\theta(1)$ and $\tau_{0}$ is a character of an irreducible projective representation of $I / N$ of degree $e$.

Lemma 2.3. [18, Lemma 3] Let $G / N$ be a solvable factor group of $G$ minimal with respect to being nonabelian. Then two cases can occur.

(a) $G / N$ is an $r$-group for some prime $r$. Hence there exists $\psi \in \operatorname{Irr}(G / N)$ such that $\psi(1)=r^{b}>1$. If $\chi \in \operatorname{Irr}(G)$ and $r \nmid \chi(1)$, then $\chi \tau \in \operatorname{Irr}(G)$ for all $\tau \in \operatorname{Irr}(G / N)$.

(b) $G / N$ is a Frobenius group with an elementary abelian Frobenius kernel $F / N$. Then $f=|G: F| \in \operatorname{cd}(G)$ and $|F / N|=r^{a}$ for some prime $r$, and a is the smallest integer such that $r^{a} \equiv 1 \bmod f$. If $\psi \in \operatorname{Irr}(F)$, then either $f \psi(1) \in \operatorname{cd}(G)$ or $r^{a}$ divides $\psi(1)^{2}$. In the latter case, $r$ divides $\psi(1)$.

(1) If no proper multiple of $f$ is in $\operatorname{cd}(G)$, then $\chi(1)$ divides $f$ for all $\chi \in$ $\operatorname{Irr}(G)$ such that $r \nmid \chi(1)$, and if $\chi \in \operatorname{Irr}(G)$ such that $\chi(1) \nmid f$, then $r^{a} \mid \chi(1)^{2}$.

(2) If $\chi \in \operatorname{Irr}(G)$ such that no proper multiple of $\chi(1)$ is in $\operatorname{cd}(G)$, then either $f$ divides $\chi(1)$ or $r^{a}$ divides $\chi(1)^{2}$. Moreover if $\chi(1)$ is divisible by no nontrivial proper character degree in $G$, then $f=\chi(1)$ or $r^{a} \mid \chi(1)^{2}$.

Lemma 2.4. Let $G$ be a finite group.

(a) If $G$ is a nonabelian simple group, then there exists a nontrivial irreducible character $\varphi$ of $G$ that extends to $\operatorname{Aut}(G)$.

(b) If $N$ is a minimal normal subgroup of $G$ so that $N \cong S^{k}$, where $S$ is a nonabelian simple group, and $\varphi \in \operatorname{Irr}(S)$ extends to $\operatorname{Aut}(S)$, then $\varphi^{k} \in \operatorname{Irr}(N)$ extends to $G$.

Proof. Part (a) follows from [4, Theorems 2-4] and to prove part (b) see [4, Lemma 5].

Lemma 2.5. [10, Lemma 6] Suppose that $M \unlhd G^{\prime}=G^{\prime \prime}$ and for every $\lambda \in \operatorname{Irr}(M)$ with $\lambda(1)=1, \lambda^{g}=\lambda$ for all $g \in G^{\prime}$. Then $M^{\prime}=\left[M, G^{\prime}\right]$ and $\left|M / M^{\prime}\right|$ divides the order of the Schur multiplier of $G^{\prime} / M$.

Lemma 2.6. [13, Theorem D] Let $N$ be a normal subgroup of a finite group $G$ and let $\varphi \in \operatorname{Irr}(N)$ be $G$-invariant. Assume that $\chi(1) / \varphi(1)$ is odd, for all $\chi(1) \in \operatorname{Irr}(G \mid \varphi)$. Then $G / N$ is solvable.

\section{Degree properties of Almost simple groups With Socle Sporadic}

In this section, we determine all finite simple groups whose irreducible character degrees divide some irreducible character degrees of almost simple groups whose socles are sporadic simple groups.

Proposition 3.1. Let $S$ be a simple group, and let $H$ be an almost simple group whose socle is a sporadic simple group. Then the character degrees of $S$ divides some character degrees of $H$ if and only if $H$ and $S$ are as in Table 1. 
Proof. Suppose that $H$ is an almost simple group with socle a sporadic simple group $H_{0}$. Suppose also that $S$ is a simple group whose degrees divide some degrees of $H$. Then the prime divisors of the degrees of $S$ are exactly those primes dividing $|S|$. Therefore, $\pi(S) \subseteq \pi(H)$, and hence by [21], we know all such possible simple groups $S$. We only need to check if the degrees of $S$ divide some degrees of $H$ and this could mainly be done by $[7,8]$. Since our arguments are similar for each group $H$, we only give a detailed proof for the cases where $H$ is $M_{12}: 2$ or $M_{22}: 2$ which will be frequently used and referred to in Sections 4 and 5 .

Suppose first $H=M_{12}: 2$. Then $\pi(S) \subseteq\{2,3,5,11\}$, and so by [21], $S$ is isomorphic to one of the simple groups $A_{5}, A_{6}, L_{2}(11), U_{5}(2), S_{4}(3), M_{11}$ and $M_{12}$. Note that $U_{5}(2)$ and $S_{4}(3)$ have degrees 220 and 81, respectively. Therefore, $S$ is isomorphic to $A_{5}, A_{6}, L_{2}(11), M_{11}$ or $M_{12}$.

Suppose now $H=M_{22}: 2$. Then $\pi(S) \subseteq\{2,3,5,7,11\}$, and so by [21], $S$ is isomorphic to one of the simple groups in $\mathcal{A}_{1} \cup \mathcal{A}_{2} \cup \mathcal{A}_{3}$, where

$$
\begin{aligned}
\mathcal{A}_{1}:= & \left\{A_{5}, A_{6}, A_{7}, L_{2}(7), L_{2}(8), M_{22}\right\}, \\
\mathcal{A}_{2}:= & \left\{A_{8}, L_{3}(4), L_{2}(49), U_{3}(3), S_{4}(7), M_{11}\right\}, \\
\mathcal{A}_{3}:= & \left\{A_{9}, A_{10}, A_{11}, A_{12}, L_{2}(11), U_{3}(5), U_{4}(3), U_{5}(2), U_{6}(2),\right. \\
& \left.S_{4}(3), S_{6}(2), O_{8}^{+}(2), M_{12}, M c L, H S, J_{2}\right\} .
\end{aligned}
$$

If $S \in \mathcal{A}_{2}$, then $S$ has a degree divisible by $25,27,44,49$ or 64 , which is a contradiction. If $S \in \mathcal{A}_{3}$, then $S$ has a degree which is divisible by 12 , which is also a contradiction. Therefore $S \in \mathcal{A}_{1}$ as claimed.

Table 1: Simple groups $S$ whose irreducible character degrees divide some character degrees of almost simple groups $H$ with socle sporadic simple groups.

\begin{tabular}{ll}
\hline \multicolumn{1}{c}{$H$} & \multicolumn{1}{c}{$S$} \\
\hline$M_{11}$ & $A_{n}$ for $n=5,6, M_{11}$ \\
$M_{12}, M_{12}: 2$ & $A_{n}$ for $n=5,6, L_{2}(11), M_{11}, M_{12}$ \\
$M_{22}, M_{22}: 2$ & $A_{n}$ for $n=5,6,7, L_{2}(q)$ for $q=7,8, M_{22}$ \\
$M_{23}$ & $A_{n}$ for $n=5,6,7, L_{2}(q)$ for $q=7,8, M_{11}, M_{23}$ \\
$M_{24}$ & $A_{n}$ for $n=5,6,7,8, L_{2}(q)$ for $q=7,8,11,23, L_{3}(4)$, \\
& $U_{3}(3), M_{11}, M_{24}$ \\
$J_{1}$ & $A_{5}, L_{2}(q)$ for $q=7,11, J_{1}$ \\
$J_{2}$ & $A_{n}$ for $n=5,6,7, L_{2}(q)$ for $q=7,8, U_{3}(3), J_{2}$ \\
$J_{2}: 2$ & $A_{8}, L_{3}(4)$, and all $S$ in $J_{2}$, \\
$J_{3}, J_{3}: 2$ & $A_{n}$ for $n=5,6, S_{4}(3), L_{2}(q)$ for $q=16,17,19, J_{3}$ \\
$J_{4}$ & $A_{n}$ for $n=5,6,7,8, L_{2}(q)$ for $q$ \\
& $7,8,11,23,29,31,32,43, L_{3}(4), L_{5}(2), U_{3}(3), U_{3}(11)$, \\
& $M_{11}, M_{12}, M_{22}, J_{4} \quad$ \\
& $A_{n}$ for $n=5,6,7,8, L_{2}(q)$ for $q=7,8,11, L_{3}(4), M_{11}$, \\
& $M_{22}, H S, H S: 2 \quad$ for $n=5,6,7,8, L_{2}(q)$ for $q=7,8,11, L_{3}(4)$, \\
$M c L, M c L: 2$ & $A_{n}$ Continud \\
& $U_{3}(3), U_{3}(5) S_{4}(3), M_{11}, M c L$ \\
\hline &
\end{tabular}


Table 1 - Continued

\begin{tabular}{|c|c|}
\hline$H$ & $S$ \\
\hline Suz, & $\begin{array}{l}A_{n} \text { for } n=5, \ldots, 10, L_{2}(q) \text { for } q=7,8,11,13,25, \\
27,64, L_{3}(3), L_{3}(4), L_{3}(9), L_{4}(3), U_{3}(3), U_{3}(4), U_{4}(2), \\
U_{4}(3), U_{5}(2), S_{6}(2),{ }^{2} B_{2}(8), G_{2}(3),{ }^{2} F_{4}(2)^{\prime}, M_{11}, M_{12}, \\
M_{22}, S u z, J_{2}\end{array}$ \\
\hline Suz : 2 & $A_{11}$, and all $S$ in $S u z$ \\
\hline $\mathrm{Co}_{3}$ & $\begin{array}{l}A_{n} \text { for } n=5,6,7,8,9,11, L_{2}(q) \text { for } q=7,8,11,23, \\
L_{3}(4), U_{3}(q) \text { for } q=3,5, U_{4}(3), S_{4}(3), S_{6}(2), M_{11}, \\
M_{12}, M_{22}, M_{23}, M_{24}, C_{3}\end{array}$ \\
\hline $\mathrm{CO}_{2}$ & $\begin{array}{l}A_{n} \text { for } n=5, \ldots, 11, L_{2}(q) \text { for } q=7,8,11,23, L_{3}(4) \text {, } \\
U_{3}(q) \text { for } q=3,5, U_{4}(3), U_{5}(2), S_{4}(3), S_{6}(2), M_{11}, \\
M_{12}, M_{22}, M_{23}, M_{24} J_{2}, C O_{2}\end{array}$ \\
\hline $\mathrm{Co}_{1}$ & $\begin{array}{l}A_{n} \text { for } n=5, \ldots, 16, L_{2}(q) \text { for } q=7,8,11,13,23, \\
25,27,49,64, L_{3}(q) \text { for } q=3,4,9, L_{4}(3), U_{3}(q) \text { for } \\
q=3,4,5, U_{4}(3), U_{5}(2), U_{6}(2), S_{4}(q) \text { for } q=3,5, \\
8, S_{6}(q) \text { for } q=2,3,{ }^{2} B_{2}(8), O_{7}(3), O_{8}^{+}(2), G_{2}(q) \text { for } \\
q=3,4,{ }^{3} D_{4}(2),{ }^{2} F_{4}(2)^{\prime}, M_{11}, M_{12}, M_{22}, M_{23}, M_{24}, \\
M c L, J_{2}, H S, C C_{1}, C_{3}\end{array}$ \\
\hline $\mathrm{He}, \mathrm{He}: 2$ & $\begin{array}{l}A_{n} \text { for } n=5,6,7,8, L_{2}(q) \text { for } q=7,8,16,17,49 \text {, } \\
L_{3}(4), U_{3}(3), S_{4}(4), H e\end{array}$ \\
\hline$F i_{22}$ & $\begin{array}{l}A_{n} \text { for } n=5, \ldots, 11, A_{13}, L_{2}(q) \text { for } q=7,8,11, \\
13,25,27,64, L_{3}(q) \text { for } q=3,4,9, L_{4}(3), U_{3}(q) \text { for } \\
q=3,4, U_{4}(3), U_{5}(2), U_{6}(2), S_{4}(3), S_{6}(2),{ }^{2} B_{2}(8), \\
O_{8}^{+}(2), G_{2}(q) \text { for } q=3,4, M_{11}, M_{12}, M_{22}, J_{2}, M c L, \\
F i_{22}\end{array}$ \\
\hline
\end{tabular}

$F i_{22}: 2 \quad S_{6}(3), O_{7}(3)$, and all $S$ in $F i_{22}$.

$F i_{23}$

$A_{n}$ for $n=5, \ldots, 13, L_{2}(q)$ for $q=7,8,11,13,16$,

$17,23,25,27,64, L_{3}(q)$ for $q=3,4,9,16^{2}, L_{4}(q)$ for $q=3,4, U_{3}(q)$ for $q=3,4, U_{4}(q)$ for $q=3$, $4, U_{5}(2), S_{4}(3), S_{4}(4), S_{6}(2), S_{6}(3),{ }^{2} B_{2}(8), O_{7}(3)$, $O_{8}^{+}(2), O_{8}^{-}(2), G_{2}(q)$ for $q=3,4,{ }^{2} F_{4}(2)^{\prime}, M_{11}, M_{12}$, $M_{22}, M_{23}, M_{24}, H S, J_{2}, F i_{23}$

$F i_{24}^{\prime}, F i_{24}^{\prime}: 2 \quad A_{n}$ for $n=5, \ldots, 14, L_{2}(q)$ for $q=7,8,11,13,16,17$, $23,25,27,29,49,64, L_{3}(q)$ for $q=3,4,9,16^{2}, L_{4}(q)$ for $q=3,4, L_{6}(3), U_{3}(q)$ for $q=3,4, U_{4}(3), U_{5}(2)$, $S_{4}(q)$ for $q=3,4,8, S_{6}(2), S_{6}(3), S_{8}(2),{ }^{2} B_{2}(8)$, $\mathrm{O}_{7}(3), O_{8}^{ \pm}(2), G_{2}(q)$ for $q=3,4,{ }^{3} D_{4}(2),{ }^{2} F_{4}(2)^{\prime}$, $M_{11}, M_{12}, M_{22}, M_{23}, M_{24}, H e, J_{2}, F i_{24}^{\prime}$

Th $A_{n}$ for $n=5, \ldots, 10, L_{2}(q)$ for $q=7,8,13,19,25$, $27,31,49,64, L_{3}(q)$ for $q=3,4,5,9, L_{4}(3), L_{5}(2)$, $U_{3}(q)$ for $q=3,4,5,8, S_{4}(3), S_{4}(8), S_{6}(2),{ }^{2} B_{2}(8)$, ${ }^{3} D_{4}(2), G_{2}(q)$ for $q=3,4,{ }^{2} F_{4}(2){ }^{\prime}, J_{2}, T h$

Ru $\quad A_{n}$ for $n=5, \ldots, 8, L_{2}(q)$ for $q=7,8,13,25,27,29$, $64, L_{3}(q)$ for $q=3,4, U_{3}(q)$ for $q=3,4,5, J_{2}, R u$ 
Table 1 - Continued

\begin{tabular}{|c|c|}
\hline$H$ & $S$ \\
\hline$L y$ & $\begin{array}{l}A_{n} \text { for } n=5, \ldots, 9, A_{11}, L_{2}(q) \text { for } q=7,8,11,31 \text {, } \\
32, L_{3}(q) \text { for } q=4,5, U_{3}(q) \text { for } q=3,5, S_{4}(3), M_{11} \text {, } \\
M_{12}, M_{22}, J_{2}, M c L, H S, L y\end{array}$ \\
\hline$H N, H N: 2$ & $\begin{array}{l}A_{n} \text { for } n=5, \ldots, 10, L_{2}(q) \text { for } q=7,8,11,19, L_{3}(q) \\
\text { for } q=4.19, L_{4}(7), U_{3}(q) \text { for } q=3,5,8, U_{4}(3) \text {, } \\
S_{4}(3), O_{8}^{+}(2), M_{11}, M_{12}, M_{22}, J_{1}, J_{2}, H S, H N\end{array}$ \\
\hline$O^{\prime} N, O^{\prime} N: 2$ & $\begin{array}{l}A_{n} \text { for } n=5, \ldots, 8, L_{2}(q) \text { for } q=7,8,11,16,17,19, \\
31,32, L_{3}(q) \text { for } q=4,7, U_{3}(q) \text { for } q=3,8, S_{4}(3) \text {, } \\
S_{6}(2), M_{11}, M_{12}, M_{22}, O^{\prime} N\end{array}$ \\
\hline$B$ & $\begin{array}{l}A_{n} \text { for } n=5, \ldots, 28, L_{2}(q) \text { for } q=7,8,11,13,16, \\
17,19,23,27,31,32,47,49,64,125, L_{3}(q) \text { for } q=3, \\
4,5,9,16,25, L_{4}(q) \text { for } q=3,4,5, L_{5}(2), L_{5}(4), \\
L_{6}(2), L_{6}(4), U_{3}(q) \text { for } q=3,4,5,8, U_{4}(q) \text { for } q=2, \\
3,4,5,8, U_{5}(2), U_{6}(2), S_{4}(q) \text { for } q=4,5,7,8, G_{2}(q) \\
\text { for } q=3,4,5, O_{7}(3), O_{8}^{ \pm}(2), O_{8}^{+}(3), O_{10}^{ \pm}(2), O_{12}^{ \pm}(2), \\
{ }^{2} F_{4}(2){ }^{\prime},{ }^{3} D_{4}(2), M_{11}, M_{12}, M_{22}, M_{23}, M_{24}, J_{1}, J_{2}, \\
J_{3}, H S, M c L, S u z, F i_{22}, C O_{3}, C o_{2}, T h, B\end{array}$ \\
\hline$M$ & $\begin{array}{l}A_{n} \text { for } n=5, \ldots, 36, L_{2}(q) \text { for } q=7,8,11,13,16, \\
17,19,23,25,27,29,31,32,41,47,49,59,64,71,81, \\
169,1024, L_{3}(q) \text { for } q=3,4,5,7,9,16,19,25, L_{4}(q) \\
\text { for } q=3,4,5,7,9, L_{5}(q) \text { for } q=2,4, L_{6}(q), \text { for } q= \\
2,3,4, U_{3}(q) \text { for } q=3,4,5,8,27, U_{4}(q) \text { for } q=2,3, \\
4,5,8,9, U_{5}(q) \text { for } q=2,4, U_{6}(2) \text { for } q=2,4, S_{4}(q) \\
\text { for } q=4,5,7,8,9, S_{6}(q) \text { for } q=2,3,4,5, S_{8}(2), \\
S_{10}(2),{ }^{2} B_{2}(8),{ }^{2} B_{2}(32), G_{2}(q) \text { for } q=3,4,5, O_{7}(3), \\
O_{7}(5), O_{9}(3), O_{8}^{ \pm}(2), O_{8}^{ \pm}(3), O_{10}^{ \pm}(2), O_{10}^{ \pm}(3), O_{12}^{ \pm}(2), \\
{ }^{2} G_{2}(27),{ }^{2} F_{4}(2){ }^{\prime}, F_{4}(2),{ }^{3} D_{4}(2), M_{11}, M_{12}, M_{22}, M_{23}, \\
M_{24}, J_{1}, J_{2}, J_{3}, H S, M c L, S u z, F i_{22}, C_{3}, C o_{2}, T h, \\
H e, O^{\prime} N, R u, M\end{array}$ \\
\hline
\end{tabular}

\section{Groups With SOCLE $M_{12}$}

In this section, we prove Theorem 1.1 for almost simple group $H$ whose socle is $H_{0}:=M_{12}$. By [11], Theorem 1.1 is proved when $H=H_{0}=M_{12}$. Therefore, we only need to deal with the case where $H:=M_{12}: 2$. For convenience, we mention some properties of $H$ and $H_{0}$ some of which can be obtained in [7, pp. 31-33].

Lemma 4.1. Let $H_{0}:=M_{12}$ and $H:=M_{12}: 2$. Then

(a) The Schur multiplier and the group of outer automorphisms of $H_{0}$ are $\mathbb{Z}_{2}$;

(b) The degrees of irreducible characters of $H$ are

\begin{tabular}{|c|c|c|c|}
\hline 1 & $45=3^{2} \cdot 5$ & $66=2 \cdot 3 \cdot 11$ & $120=2^{3} \cdot 3 \cdot 5$ \\
\hline $22=2 \cdot 11$ & $54=2 \cdot 3^{3}$ & $99=3^{2} \cdot 11$ & $144=2^{4} \cdot 3^{2}$ \\
\hline $32=2^{5}$ & $55=5 \cdot 11$ & $110=2 \cdot 5 \cdot 11$ & $176=2^{4} \cdot 11$ \\
\hline
\end{tabular}

(c) If $K$ is a maximal subgroup of $H_{0}$ whose index in $H_{0}$ divides some degrees $\chi(1)$ of $H$, then one of the following occurs: 
(i) $K \cong M_{11}$ and $\chi(1) /\left|H_{0}: K\right|$ divides $2 \cdot 5$ or $2^{2} \cdot 3$;

(ii) $K \cong M_{10}: 2$ and $\chi(1) /\left|H_{0}: K\right|=1$;

(iii) $K \cong L_{2}(11)$ and $\chi(1) /\left|H_{0}: K\right|=1$.

(d) If $S$ is a finite nonabelian simple group whose irreducible character degrees divide some degrees of $H$, then $S$ is isomorphic to $A_{5}, A_{6}, L_{2}(11), M_{11}$ or $M_{12}$.

Proof. Parts (a) and (b) follows from [7, pp. 31-33] and part (d) follows from Proposition 3.1 and Table 1. Part (c) is a straightforward calculation.

4.1. Proof of Theorem 1.1 for $M_{12}: 2$. As noted before, by [11], we may assume that $H:=M_{12}: 2$. We further assume that $G$ is a finite $\operatorname{group}$ with $\operatorname{cd}(G)=$ $\operatorname{cd}\left(M_{12}: 2\right)$. The proof of Theorem 1.1 follows from the following lemmas.

Lemma 4.2. $G^{\prime}=G^{\prime \prime}$.

Proof. Assume the contrary. Then there is a normal subgroup $N$ of $G$, where $N$ is maximal such that $G / N$ is a nonabelian solvable group. Now we apply Lemma 2.3 and we have one of the following cases:

(a) $G / N$ is a $r$-group with $r$ prime. In this case, $G / N$ has an irreducible character $\psi$ of degree $r^{b}>1$, and so does $G$. Since $M_{12}: 2$ has an irreducible character of degree 32 , we conclude that $r=2$. Let now $\chi \in \operatorname{Irr}(G)$ with $\chi(1)=99$. Then Lemma 2.1(a) implies that $\chi_{N} \in \operatorname{Irr}(N)$, and so by Lemma 2.1(b), $G$ has an irreducible character of degree $99 \psi(1)$, which is a contradiction.

(b) $G / N$ is a Frobenius group with kernel $F / N$. Then $|G: F| \in \operatorname{cd}(G)$ divides $r^{a}-1$, where $|F / N|=r^{a}$. Let $|G: F| \in\{2 \cdot 11,5 \cdot 11\}$ and $\chi(1)=2^{4} \cdot 3^{2}$. Then Lemma 2.2(b) implies that $r^{a}$ divides $\chi^{2}(1)=2^{8} \cdot 3^{4}$, and it is impossible as $|G: F|$ does not divide $r^{a}-1$, for every divisor $r^{a}$ of $2^{8} \cdot 3^{4}$. Let now $|G: F| \notin\{2 \cdot 11,5 \cdot 11\}$. Then no proper multiple of $|G: F|$ is in $\operatorname{cd}(G)$. If $r=2$, then by Lemma 2.2(a), both $3^{2} \cdot 11$ and $3^{2} \cdot 5$ must divide $|G: F|$, which is a contradiction. If $r=3$, then by Lemma $2.2(\mathrm{a}),|G: F|$ is divisible by $2^{5}$ and $2^{4} \cdot 11$, which is a contradiction. Similarly, if $r \neq 2,3$, then $2^{5}$ and $2^{4} \cdot 3^{2}$ divide $|G: F|$, which is a contradiction.

Lemma 4.3. Let $G^{\prime} / M$ be a chief factor of $G$. Then $G^{\prime} / M \cong M_{12}$.

Proof. Suppose $G^{\prime} / M \cong S^{k}$, where $S$ is a nonabelian simple group for some positive integer $k$. Since $S$ is a finite nonabelian simple group whose irreducible character degrees divide some degrees of $M_{12}: 2$, by Lemma 4.1(d), $S$ is isomorphic to one of the groups $A_{5}, A_{6}, M_{11}, M_{12}$ or $L_{2}(11)$. If $S$ is isomorphic to one of the groups $A_{5}$, $A_{6}, M_{11}, M_{12}, L_{2}(11)$, then $k=1$ as $G$ has no degree divisible by $5^{2}$. Assume that $S$ is isomorphic to $A_{5}$ or $A_{6}$, then $G^{\prime} / M$ has a character $\psi$ of degree 5 . If $\chi$ is an irreducible constituent of $\psi^{G / M}$, then $\chi(1)=t \psi(1)=5 t$, where $t$ divides $|\operatorname{Out}(S)|$. Consequently, $G$ has a character of degree at most 20 , which is a contradiction. Similarly, in the case where $S$ is isomorphic to $M_{11}$ or $L_{2}(11)$, the factor group $G / M$ has a character of degree $10 t$ with $t=1,2$, and this implies that $G$ has a character of degree at most 20 , which is a contradiction. Therefore $G^{\prime} / M$ is isomorphic to $M_{12}$.

Lemma 4.4. Let $\theta \in \operatorname{Irr}(M)$ with $\theta(1)=1$. Then $I_{G^{\prime}}(\theta)=G^{\prime}$ and $M=M^{\prime}$.

Proof. Suppose $I=I_{G^{\prime}}(\theta)<G^{\prime}$. By Lemma 2.2, we have $\theta^{I}=\sum_{i=1}^{k} \phi_{i}$ where $\phi_{i} \in$ $\operatorname{Irr}(I)$ for $i=1,2, \ldots, k$. Let $U / M$ be a maximal subgroup of $G^{\prime} / M \cong M_{12}$ containing 
$I / M$ and set $t:=|U: I|$. It follows from Lemma 2.2(a) that $\phi_{i}(1)\left|G^{\prime}: I\right| \in \operatorname{cd}\left(G^{\prime}\right)$, and so $t \phi_{i}(1)\left|G^{\prime}: U\right|$ divides some degrees of $G$. Then $\left|G^{\prime}: U\right|$ must divide some character degrees of $G$, and hence by Lemma 4.1(c) one of the following holds.

(i) Suppose $U / M \cong M_{11}$. Then $t \phi_{i}(1)$ divide $2 \cdot 5$ or $2^{2} \cdot 3$. If $t=1$, then $I / M \cong M_{11}$. Since $M_{11}$ has trivial Schur multiplier, it follows that $\theta$ extends to $\theta_{0} \in \operatorname{Irr}(I)$, and so by Lemma 2.2(b) $\left(\theta_{0} \tau\right)^{G^{\prime}} \in \operatorname{Irr}\left(G^{\prime}\right)$, for all $\tau \in \operatorname{Irr}(I / M)$. For $\tau(1)=55 \in \operatorname{cd}\left(M_{11}\right)$, it turns out that $12 \cdot 55 \cdot \theta_{0}(1)$ divide some degrees of $G$, which is a contradiction. Therefore, $t \neq 1$, and hence the index of a maximal subgroup of $U / M \cong M_{11}$ containing $I / M$ must divide $2 \cdot 5$ or $2^{2} \cdot 3$. This implies that $t \phi_{i}(1)$ divides $2^{2} \cdot 3$ and $I / M \cong L_{2}(11)$. In particular, $\phi_{i}(1)=1$. Thus $\theta$ extends to a $\phi_{i}$, and so by Lemma $2.2(\mathrm{~b}), 144 \tau(1) \in \operatorname{cd}\left(G^{\prime}\right)$, for all $\tau \in \operatorname{Irr}(I / M)$. This leads us to a contradiction by taking $\tau(1)=10 \in \operatorname{cd}\left(L_{2}(11)\right)$.

(ii) Suppose $U / M \cong M_{10}: 2$. In this case $t=1$, or equivalently, $I / M=U / M \cong$ $M_{10}$ : 2. Moreover, $\phi_{i}(1)=1$, for all $i$. Then $\theta$ extends to $\phi_{i} \in \operatorname{Irr}(I)$, and so by Lemma 2.2(b), $66 \tau(1)$ divides some degrees of $G$, for $\tau(1)=10$, which is a contradiction.

(iii) Suppose $U / M \cong L_{2}(11)$ and $t=\phi_{i}(1)=1$, for all $i$. Then $I / M \cong L_{2}(11)$, and so $\theta$ extends to $\phi_{i} \in \operatorname{Irr}(I)$. Thus $144 \tau(1) \in \operatorname{Irr}\left(G^{\prime}\right)$, for all $\tau \in \operatorname{Irr}(I / M)$. This is impossible by taking $\tau(1)=10$.

Therefore, $I_{G^{\prime}}(\theta)=G^{\prime}$. By Lemma 2.5, we have that $\left|M / M^{\prime}\right|$ divides the order of Schur multiplier of $G^{\prime} / M \cong M_{12}$ which is 2 . If $\left|M / M^{\prime}\right|=2$, then $G^{\prime} / M^{\prime}$ is isomorphic to $2 \cdot M_{12}$ which has a character of degree 32 [7, p. 33]. Therefore $M_{12}$ must have a degree divisible by 32 , which is a contradiction. Hence $\left|M / M^{\prime}\right|=1$, or equivalently, $M=M^{\prime}$.

Lemma 4.5. The subgroup $M$ is trivial, and hence $G^{\prime} \cong M_{12}$.

Proof. By Lemmas 4.3 and 4.4, we have that $G^{\prime} / M \cong M_{12}$ and $M=M^{\prime}$. Suppose that $M$ is nonabelian, and let $N \leqslant M$ be a normal subgroup of $G^{\prime}$ such that $M / N$ is a chief factor of $G^{\prime}$. Then $M / N \cong S^{k}$, for some nonabelian simple group $S$. It follows from Lemma 2.4 that $S$ possesses a nontrivial irreducible character $\varphi$ such that $\varphi^{k} \in \operatorname{Irr}(M / N)$ extends to $G^{\prime} / N$. By Lemma 2.1(b), we must have $\varphi(1)^{k} \tau(1) \in$ $\operatorname{cd}\left(G^{\prime} / N\right) \subseteq \operatorname{cd}\left(G^{\prime}\right)$, for all $\tau \in \operatorname{Irr}\left(G^{\prime} / M\right)$. Now we can choose $\tau \in G^{\prime} / M$ such that $\tau(1)$ is the largest degree of $M_{12}$, and since $\varphi$ is nontrivial, $\varphi(1)^{k} \tau(1)$ divides no degree of $G$, which is a contradiction. Therefore, $M$ is abelian, and since $M=M^{\prime}$, we conclude that $M=1$.

Lemma 4.6. There exists an abelian group $A$ such that $G / A \cong M_{12}: 2$.

Proof. Set $A:=C_{G}\left(G^{\prime}\right)$. Since $G^{\prime} \cap A=1$ and $G^{\prime} A \cong G^{\prime} \times A$, it follows that $G^{\prime} \cong G^{\prime} A / A \unlhd G / A \leqslant \operatorname{Aut}\left(G^{\prime}\right)$. By Lemma 4.5 , we have $G^{\prime} \cong M_{12}$, and so we conclude that $G / A$ is isomorphic to $M_{12}$ or $M_{12}: 2$. In the case where $G / A$ is isomorphic to $M_{12}$, we must have $G \cong A \times M_{12}$. This is impossible as $32 \in \operatorname{cd}(G)$ but $M_{12}$ has no character of degree 32 . Therefore, $G / A$ is isomorphic to $M_{12}: 2$.

\section{Groups With SOCLE $M_{22}$}

In this section, we prove Theorem 1.1 for almost simple group $H$ whose socle is $H_{0}:=M_{22}$. Note that Theorem 1.1 is proved for $H=H_{0}=M_{22}$, see [11]. Therefore, we only need to focus on case where $H:=M_{22}: 2$. For convenience, we mention some properties of $H$ and $H_{0}$ some of which can be obtained from [7, pp. 39-41]. 
Lemma 5.1. Let $H_{0}:=M_{22}$ and $H:=M_{22}: 2$. Then

(a) The Schur multiplier $H_{0}$ is $\mathbb{Z}_{12}$ and the group of outer automorphisms of $H_{0}$ is $\mathbb{Z}_{2}$;

(b) The degrees of irreducible characters of $H$ are

\begin{tabular}{|c|c|c|c|}
\hline 1 & $55=5 \cdot 11$ & $210=2 \cdot 3 \cdot 5 \cdot 7$ & $385=5 \cdot 7 \cdot 11$ \\
\hline $1=3 \cdot 7$ & $99=3^{2} \cdot 11$ & $231=3 \cdot 7 \cdot 11$ & $560=2^{4} \cdot 5$ \\
\hline$=3^{2} \cdot 5$ & $154=2 \cdot 7 \cdot 1$ & & \\
\hline
\end{tabular}

(c) If $K$ is a maximal subgroup of $H_{0}$ whose index in $H_{0}$ divides some degrees $\chi(1)$ of $H$, then one of the following occurs:

(i) $K \cong L_{3}(4)$ and $\chi(1) /\left|H_{0}: K\right|$ divides 7 ;

(ii) $K \cong 2^{4}: S_{5}$ and $\chi(1) /\left|H_{0}: K\right|=1$;

(iii) $K \cong 2^{4}: A_{6}$ and $\chi(1) /\left|H_{0}: K\right|$ divides 2,3 or 5 .

(d) If $S$ is a finite nonabelian simple group whose irreducible character degrees divide some degrees of $H$, then $S$ is isomorphic to $A_{5}, A_{6}, A_{7}, L_{2}(7), L_{2}(8)$ or $M_{22}$.

Proof. Parts (a)-(b) follows from [7, pp. 31-33] part (d) follows from Proposition 3.1 and Table 1. Part (c) is a straightforward calculation.

5.1. Proof of Theorem 1.1 for $M_{22}: 2$. Theorem 1.1 is true for the Mathieu group $M_{22}$ by [11]. It remains to assume that $H:=M_{22}: 2$. In what follows assume that $G$ is a finite group with $\operatorname{cd}(G)=\operatorname{cd}\left(M_{22}: 2\right)$. The proof follows from the following lemmas.

Lemma 5.2. $G^{\prime}=G^{\prime \prime}$.

Proof. Assume the contrary. Then there is a normal subgroup $N$ of $G$ where $N$ is a maximal such that $G / N$ is a nonabelian solvable group. We now apply Lemma 2.3, and so we have the following two cases:

(a) Suppose that $G / N$ is a $r$-group with $r$ prime. Then it has an irreducible character $\psi$ of degree $r^{b}>1$. It is impossible as the group $M_{22}: 2$ does not have a irreducible character of prime power degree.

(b) $G / N$ is a Frobenius group with kernel $F / N$. Then $|G: F| \in \operatorname{cd}(G)$ divides $r^{a}-1$, where $|F / N|=r^{a}$. Let $|G: F| \in\{3 \cdot 7,5 \cdot 11\}$ and $\chi(1)=2 \cdot 7 \cdot 11$. Then Lemma 2.2(b) implies that $r^{a}$ divides $\chi^{2}(1)=2^{2} \cdot 7^{2} \cdot 11^{2}$, and it is impossible as $|G: F|$ does not divide $r^{a}-1$, for every divisor $r^{a}$ of $2^{2} \cdot 7^{2} \cdot 11^{2}$. Let now $|G: F| \notin\{3 \cdot 7,5 \cdot 11\}$. Then no proper multiple of $|G: F|$ is in $\operatorname{cd}(G)$. If $r=3$, then by Lemma 2.2(a), both $5 \cdot 7 \cdot 11$ and $2^{4} \cdot 5 \cdot 7$ must divide $|G: F|$, which is a contradiction. If $r=5$, then by Lemma $2.2(\mathrm{a})$, both $3 \cdot 7 \cdot 11$ and $2 \cdot 7 \cdot 11$ must divide $|G: F|$, which is a contradiction. If $r=11$, then by Lemma $2.2(\mathrm{a}),|G: F|$ is divisible by $2 \cdot 3 \cdot 5 \cdot 7$ and $2^{4} \cdot 5 \cdot 7$, which is a contradiction. Similarly, if $r \neq 3,5$, and 11 , then $3^{2} \cdot 11$ and $5 \cdot 11$ divide $|G: F|$, which is impossible. Therefore, $G^{\prime}=G^{\prime \prime}$.

Lemma 5.3. Let $G^{\prime} / M$ be a chief factor of $G$. Then $G^{\prime} / M \cong M_{22}$.

Proof. Suppose $G^{\prime} / M \cong S^{k}$, where $S$ is a nonabelian simple group and for some positive integer $k$. Since $S$ is a finite nonabelian simple group whose irreducible character degrees divide some degrees of $M_{22}: 2$, by Lemma $5.1(\mathrm{~d})$, the group $S$ is isomorphic to $A_{5}, A_{6}, A_{7}, L_{2}(7), L_{2}(8)$ or $M_{22}$. Observe that $G$ has no degree 
TABLE 2. The triples $(S, \psi, d)$ in Lemma 5.3

\begin{tabular}{lccc}
\hline$S$ & Out $(S)$ & $\psi(1)$ & $d$ \\
\hline$A_{5}$ & 2 & 5 & 10 \\
$A_{6}$ & 4 & 5 & 20 \\
$A_{7}, L_{2}(7)$ & 2 & 6 & 12 \\
$L_{2}(8)$ & 3 & 8 & 24 \\
\hline
\end{tabular}

divisible by 25 and 49 . This implies that $k=1$ in each case. Assume that $S$ is isomorphic to one of the simple groups as in the first column of Table 2. Then by [7], $G^{\prime} / M$ has a character $\psi$ of degree as in the third column of the same Table 2 . If $\chi$ is an irreducible constituent of $\psi^{G / M}$, then $\chi(1)=t \psi(1)$, where $t$ divides $|\operatorname{Out}(S)|$. Consequently, $G$ has a character of degree at most $d$ as in the forth column of Table 2, which is a contradiction. For example, if $S$ is isomorphic to $A_{7}$ or $L_{2}(7)$, then $G^{\prime} / M$ has a character $\psi$ of degree 6 , then $\chi(1)=t \psi(1)=6 t$, where $t$ divides $|\operatorname{Out}(S)|$, and so $G$ has a character of degree at most 12 , which is a contradiction. Therefore $G^{\prime} / M \cong M_{22}$.

Lemma 5.4. If $\theta \in \operatorname{Irr}(M)$, then $I_{G^{\prime}}(\theta)=G^{\prime}$ and $M=M^{\prime}$.

Proof. Suppose $I:=I_{G^{\prime}}(\theta)<G^{\prime}$. By Lemma 2.2 we have $\theta^{I}=\sum_{i=1}^{k} \phi_{i}$ where $\phi_{i} \in$ $\operatorname{Irr}(I)$ for $i=1,2, \ldots, k$. Let $U / M$ be a maximal subgroup of $G^{\prime} / M \cong M_{22}$ containing $I / M$ and set $t:=|U: I|$. It follows from Lemma 2.2(a) that $\phi_{i}(1)\left|G^{\prime}: I\right| \in \operatorname{cd}\left(G^{\prime}\right)$, and so $t \phi_{i}(1)\left|G^{\prime}: U\right|$ divides some degrees of $G$. Then $\left|G^{\prime}: U\right|$ must divide some character degrees of $G$, and hence by Lemma 5.1 one of the following holds:

(i) Suppose $U / M \cong L_{3}(4)$. Then, for each $i, t \phi_{i}(1)$ divide 7 . As $U / M \cong L_{3}(4)$ does not have any subgroup of index 7 , by [7, p. 23], so $t=1$ and $I / M \cong U / M \cong L_{3}(4)$ and $\phi_{i}(1)$ divide 7 . If $\phi_{i}(1)=1$, then $\theta$ extend to $\phi_{i}$, and so by Lemma 2.2(b), $\left(\phi_{i} \tau\right)^{G^{\prime}} \in \operatorname{Irr}\left(G^{\prime}\right)$, for all $\tau \in \operatorname{Irr}(I / M)$. If $\tau(1)=64 \in \operatorname{cd}\left(L_{3}(4)\right)$, then $22 \tau(1)=2^{7} \cdot 11$ divides some degrees of $G$, which is a contradiction. Hence $\phi_{i}(1)=7$, for all $i$. Then $\phi_{i_{M}}=e_{i} \theta$, where $e_{i} \neq 1$ is the degree of a projective representation of $I / M \cong L_{3}(4)$, and it is impossible by [7, p. 24].

(ii) Suppose $U / M \cong 2^{4}: S_{5}$. Then $I / M \cong 2^{4}: S_{5}$ and $\phi_{i}(1)=1$, for all $i$. Thus $\theta$ extends to $\phi_{i}$ in $I$. It follows from Lemma $2.2(\mathrm{~b})$ that $\tau(1)\left|G^{\prime}: I\right|$ divides some character degrees of $G$, for all $\tau \in I / M$. This is impossible by taking $\tau(1)=4$.

(iii) Suppose $U / M \cong 2^{4}: A_{6}$. In this case, $t \phi_{i}(1)$ divides 2,3 or 5 . It follows from [6] that $U / M$ has no maximal subgroup of index 2, 3 and 5, and this implies that $I=U$. Therefore, $I / M \cong 2^{4}: A_{6}$, that is to say, $I / M$ has an abelian subgroup $A / M$ of order $2^{4}$ such that $I / A \cong A_{6}$.

Let now $\lambda \in \operatorname{Irr}(A \mid \theta)$, and write $\lambda^{I}=\sum f_{i} \mu_{i}$. Since $A \unlhd I$, the degree $\mu_{i}(1)$ divides 2,3 or 5 , for all $i$. Since the index of a maximal subgroup of $I / A \cong A_{6}$ is at least $6, \lambda$ is $I$-invariant, and so $\mu_{i_{A}}=f_{i} \lambda$, for all $i$. If $f_{i}=1$ for some $i$, then $\lambda$ extends to $\lambda_{0} \in \operatorname{Irr}(I)$, and so by Lemma 2.2(b), $\lambda_{0} \tau$ is an irreducible character of $\lambda^{I}$, for all $\tau \in \operatorname{Irr}(I / A)$, and so $\lambda_{0}(1) \tau(1)=\tau(1)$ divides 2,3 , or 5 . This is impossible as we could take $\tau(1)=8 \in \operatorname{cd}\left(A_{6}\right)$. Therefore, $f_{i}>1$, for all $i$. Moreover, we know from Lemma 2.2(c) that each $f_{i}$ is the degree of a nontrivial proper irreducible projective representation of $A_{6}$, by [7, p. 5], we observe that $f_{i} \in\{3,5\}$. This shows that $\mu(1) / \lambda(1)$ is odd, for all $\mu \in \operatorname{Irr}(I \mid A)$, and so by Lemma 2.6, the group $I / A \cong A_{6}$ is solvable, which is a contradiction. 
TABlE 3. Some character degrees of $G^{\prime} / M^{\prime}$ in Lemma 5.4

\begin{tabular}{cccccc}
\hline$G^{\prime} / M^{\prime}$ & $2 \cdot M_{22}$ & $3 \cdot M_{22}$ & $4 \cdot M_{22}$ & $6 \cdot M_{22}$ & $12 \cdot M_{22}$ \\
\hline Degree & 440 & 384 & 440 & 384 & 384 \\
\hline
\end{tabular}

This show $I_{G^{\prime}}(\theta)=G^{\prime}$. By Lemma 2.5, we have that $\left|M / M^{\prime}\right|$ divides the order of Schur multiplier of $G^{\prime} / M \cong M_{22}$ which is 12 . If $\left|M / M^{\prime}\right| \neq 1$, then $G$ has an irreducible character of degree divisible by one the degrees in the second row of Table 3, which is a contradiction. Therefore, $M=M^{\prime}$.

Lemma 5.5. The subgroup $M$ is trivial, and hence $G^{\prime} \cong M_{22}$.

Proof. It follows from Lemmas 5.3 and 5.4 that $G^{\prime} / M \cong M_{22}$ and $M=M^{\prime}$. Assume that $M$ is nonabelian, and let $N \leqslant M$ be a normal subgroup of $G^{\prime}$ such that $M / N$ is a chief factor of $G^{\prime}$. Then $M / N \cong S^{k}$ for some nonabelian simple group $S$. By Lemma 2.4, $S$ has a nontrivial irreducible character $\varphi$ such that $\varphi^{k} \in \operatorname{Irr}(M / N)$ extends to $G^{\prime} / N$. Now Lemma 2.1(b) implies that $\varphi(1)^{k} \tau(1) \in \operatorname{cd}\left(G^{\prime} / N\right) \subseteq \operatorname{cd}\left(G^{\prime}\right)$, for all $\tau \in \operatorname{Irr}\left(G^{\prime} / M\right)$. As $\varphi(1)>1$, if we choose $\tau \in G^{\prime} / M$ such that $\tau(1)$ is the largest degree of $M_{22}$, then $\varphi(1)^{k} \tau(1)$ divides no degree of $G$, which is a contradiction. Therefore, $M$ is abelian, and hence we are done.

Lemma 5.6. There exists an abelian group $A$ such that $G / A \cong M_{22}: 2$.

Proof. Set $A:=C_{G}\left(G^{\prime}\right)$. Since $G^{\prime} \cap A=1$ and $G^{\prime} A \cong G^{\prime} \times A$, it follows that $G^{\prime} \cong G^{\prime} A / A \unlhd G / A \leqslant \operatorname{Aut}\left(G^{\prime}\right)$. Since $G^{\prime} \cong M_{22}$, we conclude that $G / A$ is isomorphic to $M_{22}$ or $M_{22}: 2$. In the case where $G / A$ is isomorphic to $M_{22}$, we conclude that $G \cong A \times M_{22}$. This is impossible as $560 \in \operatorname{cd}(G)$ but $M_{22}$ has no character of degree 560. Therefore, $G / A$ is isomorphic to $M_{22}: 2$.

\section{REFERENCES}

[1] S. H. Alavi, A. Daneshkah, H. P. Tong-Viet, and T. P. Wakefield. Huppert's conjecture for Fi $i_{23}$. Rend. Semin. Mat. Univ. Padova, 126:201-211, 2011. 2

[2] S. H. Alavi, A. Daneshkhah, H. P. Tong-Viet, and T. P. Wakefield. On Huppert's conjecture for the Conway and Fischer families of sporadic simple groups. J. Aust. Math. Soc., 94(3):289303, 2013. 2

[3] C. Bessenrodt, H. N. Nguyen, J. B. Olsson, and H. P. Tong-Viet. Complex group algebras of the double covers of the symmetric and alternating groups. Algebra Number Theory, 9(3):601628, 2015. 1

[4] M. Bianchi, D. Chillag, M. L. Lewis, and E. Pacifici. Character degree graphs that are complete graphs. Proc. Amer. Math. Soc., 135(3):671-676 (electronic), 2007. 3

[5] R. Brauer. Representations of finite groups. In Lectures on Modern Mathematics, Vol. I, pages 133-175. Wiley, New York, 1963. 1

[6] T. Connor and D. Leemans. An Atlas of subgroup lattices of finite almost simple groups. ArXiv e-prints, June 2013. 10

[7] J. H. Conway, R. T. Curtis, S. P. Norton, R. A. Parker, and R. A. Wilson. Atlas of finite groups. Oxford University Press, Eynsham, 1985. Maximal subgroups and ordinary characters for simple groups, With computational assistance from J. G. Thackray. 4, 6, 7, 8, 9, 10

[8] The GAP Group. GAP - Groups, Algorithms, and Programming, Version 4.7.8, 2015. 4

[9] B. Huppert. Character theory of finite groups, volume 25 of de Gruyter Expositions in Mathematics. Walter de Gruyter \& Co., Berlin, 1998. 2, 3

[10] B. Huppert. Some simple groups which are determined by the set of their character degrees. I. Illinois J. Math., 44(4):828-842, 2000. 1, 2, 3 
[11] B. Huppert. Some simple groups which are determined by the set of their character degrees i. Preprint, 2000. 2, 6, 7, 8, 9

[12] I. M. Isaacs. Character theory of finite groups. AMS Chelsea Publishing, Providence, RI, 2006. Corrected reprint of the 1976 original [Academic Press, New York; MR0460423]. 2

[13] A. Moretó. An answer to a question of Isaacs on character degree graphs. Adv. Math., 201(1):90-101, 2006. 3

[14] G. Navarro. The set of character degrees of a finite group does not determine its solvability. Proc. Amer. Math. Soc., 143(3):989-990, 2015. 1

[15] G. Navarro and N. Rizo. Nilpotent and perfect groups with the same set of character degrees. J. Algebra Appl., 13(8):1450061, 3, 2014. 1

[16] H. N. Nguyen, H. P. Tong-Viet, and T. P. Wakefield. Projective special linear groups $\operatorname{PSL}_{4}(q)$ are determined by the set of their character degrees. J. Algebra Appl., 11(6):1250108, 26, 2012. 2

[17] H. P. Tong-Viet. Alternating and sporadic simple groups are determined by their character degrees. Algebr. Represent. Theory, 15(2):379-389, 2012. 2

[18] H. P. Tong-Viet and T. P. Wakefield. On Huppert's conjecture for the Monster and Baby Monster. Monatsh. Math., 167(3-4):589-600, 2012. 2, 3

[19] T. P. Wakefield. Verifying Huppert's conjecture for $\operatorname{PSL}_{3}(q)$ and $\operatorname{PSU}_{3}\left(q^{2}\right)$. Comm. Algebra, 37(8):2887-2906, 2009. 2

[20] T. P. Wakefield. Verifying Huppert's conjecture for ${ }^{2} G_{2}\left(q^{2}\right)$. Algebr. Represent. Theory, 14(4):609-623, 2011. 2

[21] A. V. Zavarnitsine. Finite simple groups with narrow prime spectrum. ArXiv e-prints, Oct. 2008. 4

S.H. Alavi, Department of Mathematics, Faculty of Science, Bu-Ali Sina UniverSITY, HAMEDAN, IRAN

E-mail address: alavi.s.hassan@basu.ac.ir alavi.s.hassan@gmail.com (Gmail is preferred)

A. Daneshkhah, Department of Mathematics, Faculty of Science, Bu-Ali Sina UNIVERSITY, HAMEDAN, IRAN

E-mail address: adanesh@basu.ac.ir daneshkhah.ashraf@gmail.com (Gmail is preferred)

A. Jafari, Department of Mathematics, Faculty of Science, Bu-Ali Sina UniverSITY, Hamedan, Iran

E-mail address: a.jaefary@gmail.com 